\title{
Characterization of slurry systems by ultrasonic techniques
}

\author{
V. Stolojanu, A. Prakash* \\ Department of Chemical and Biochemical Engineering, The University of Western Ontario, London, Ont., Canada N6A 5B9
}

Received 14 April 2000; received in revised form 30 August 2000; accepted 30 August 2000

\begin{abstract}
Ultrasonic sensors have been used to measure particle concentrations and variations in their size distribution in dense liquid-solid suspensions. The measurements are based on variations in velocity, attenuation and mean frequency of the acoustic pulse. Solid loading of up to $45 \mathrm{vol} \%$ and particle sizes of 35,70 and $180 \mu \mathrm{m}$ have been used. The observed relationships between the measured parameters and solids concentration are presented. Available theoretical approaches have been reviewed and their applications and limitations have been pointed out for the data of this study. The variation of acoustic velocity and attenuation as a function of slurry concentration are well predicted by the theory for $35 \mu \mathrm{m}$ particles but not for larger particles. Approaches for determining particle size variations in suspensions are presented. (C) 2001 Elsevier Science B.V. All rights reserved.
\end{abstract}

Keywords: Slurry; Ultrasonic; Acoustic velocity; Solids concentration; Attenuation; Peak frequency

\section{Introduction}

Multiphase particulate systems are commonly used in chemical and biochemical industry in such operations as catalytic reactions, mixing, suspension, crystallization, etc. The efficient operation of catalytic reactors would depend on hydrodynamics, heat and mass transfer rates as well as composition and distribution of catalyst particles. For phase holdup measurements several techniques have been reported in literature. These include the static-pressure techniques [1], electrical-probe techniques [2], shutter techniques [3] and optical-probe techniques [4]. However, the accuracy of static-pressure technique decreases when the solid and liquid densities are close, while optical and electrical methods require the presence of specific properties of the medium such as transparency or electrical conductivity.

Ultrasonic techniques offer several desirable characteristics such as non-intrusiveness, fast response for on-line measurement and control and potential for application at conditions of high pressure and temperature as well as in reactors with radioactive content [5]. The ultrasound signal can penetrate through the walls of a vessel offering the advantage of a truly non-invasive and non-destructive technique. An ultrasonic pulse of certain energy emitted from a vibrator (transducer) into a medium propagates through this

\footnotetext{
* Corresponding author. Tel.: +1-519-661-2111, ext. 88528; fax: +1-519-661-3498.

E-mail address: aprakas2@julian.uwo.ca (A. Prakash).
}

medium and reaches the receiver at a lower energy after a certain amount of time. The variations of solid and/or gas bubble concentration in a liquid will change the density as well as the compressibility of the medium and thus the speed and the attenuation of acoustic signal.

An accurate measurement of the variations in velocity and attenuation of the acoustic wave will indicate the make of the mixture. When the particle sizes are smaller near the wavelength of the signal or the non-dimensional acoustic wavenumber $k r \ll 1$ a simple phenomenological approach by Urick [6] may be successfully applied. The acoustic velocity is calculated with averaged values of density and compressibility of the medium components. Ament [7] improved Urick's equation by taking into account the effect of viscous boundary layer thickness and particle sizes in a more complex equation for effective density. From Ament's effective density equation, an increase in particle size in a liquid-solid system will cause a small increase in ultrasound speed for a given solid concentration and ultrasound frequency.

Compared to acoustic velocity, which is mainly dependent on the density and compressibility of the medium, the loss of energy or transmission loss is caused by many other factors, i.e. scattering, absorption, reflection, refraction, diffraction or interference. For particles smaller than pulse wavelength, scattering phenomena predominates while for larger particles, reflection and refraction phenomena at the liquid/solid interface need to be considered. A theoretical approach is discussed, where scattering, viscous and thermal losses are considered to be based on literature studies [8-11]. 


\begin{tabular}{|c|c|}
\hline \multicolumn{2}{|c|}{ Nomenclature } \\
\hline$A_{i} / A_{o i}$ & $\begin{array}{l}\text { ratio of received signal amplitude before } \\
\text { and after solids addition }\end{array}$ \\
\hline$B$ & bulk elasticity modulus $(\mathrm{Pa})$ \\
\hline$k$ & ultrasound wavenumber $\left(\mathrm{m}^{-1}\right)$ \\
\hline$k_{1}$ & complex wave vector \\
\hline$L$ & distance between transducers $(\mathrm{m})$ \\
\hline$n$ & $\begin{array}{l}\text { number of incremental particles in addition } \\
\text { to suspension }\end{array}$ \\
\hline$r$ & particle radius $(\mathrm{m})$ \\
\hline$r_{1}$ & large particles radius in binary mixture (m) \\
\hline$r_{2}$ & small particles radius in binary mixture (m) \\
\hline$r_{\text {avg }}$ & $\begin{array}{l}\text { average particle radius as defined } \\
\text { by Eq. }(7)(\mathrm{m})\end{array}$ \\
\hline$V$ & acoustic velocity in suspension $(\mathrm{m} / \mathrm{s})$ \\
\hline$V_{0}$ & acoustic velocity in solid free liquid $(\mathrm{m} / \mathrm{s})$ \\
\hline \multicolumn{2}{|c|}{ Greek letters } \\
\hline$\alpha$ & attenuation coefficient $\left(\mathrm{m}^{-1}\right)$ \\
\hline$\alpha_{\mathrm{i}}$ & $\begin{array}{l}\text { incremental attenuation with solids } \\
\text { addition }\left(\mathrm{m}^{-1}\right)\end{array}$ \\
\hline$\alpha_{\mathrm{P}}$ & total attenuation defined by Eq. (9) $\left(\mathrm{m}^{-1}\right)$ \\
\hline$\alpha_{\mathrm{S}}$ & attenuation due to scattering $\left(\mathrm{m}^{-1}\right)$ \\
\hline$\alpha_{\mathrm{V}}$ & $\begin{array}{l}\text { attenuation due to viscous drag at } \\
\text { particle surface }\left(\mathrm{m}^{-1}\right)\end{array}$ \\
\hline$\Delta \alpha_{\mathrm{V}}$ & attenuation correction for larger $k r\left(\mathrm{~m}^{-1}\right)$ \\
\hline$\beta_{1}, \beta_{2}$ & coefficients in Eq. (6) \\
\hline$\beta_{\text {eff }}$ & $\begin{array}{l}\text { effective compressibility of the } \\
\text { medium }\left(\mathrm{Pa}^{-1}\right)\end{array}$ \\
\hline$\beta_{\mathrm{f}}$ & fluid compressibility $\left(\mathrm{Pa}^{-1}\right)$ \\
\hline$\beta_{\mathrm{s}}$ & particle compressibility $\left(\mathrm{Pa}^{-1}\right)$ \\
\hline$\delta$ & ratio of fluid density to solids density $\left(\rho_{\mathrm{f}} / \rho_{s}\right)$ \\
\hline$\eta$ & fluid viscosity $(\mathrm{kg} / \mathrm{m} \mathrm{s})$ \\
\hline$\lambda$ & acoustic wavelength (m) \\
\hline$\rho_{\text {eff }}$ & effective slurry density $\left(\mathrm{kg} / \mathrm{m}^{3}\right)$ \\
\hline$\rho_{\mathrm{f}}$ & fluid density $\left(\mathrm{kg} / \mathrm{m}^{3}\right)$ \\
\hline$\rho_{\mathrm{s}}$ & particle density $\left(\mathrm{kg} / \mathrm{m}^{3}\right)$ \\
\hline$\varphi$ & $\begin{array}{l}\text { volume fraction of large particles in } \\
\text { binary mixture of solids }(-)\end{array}$ \\
\hline$\phi$ & solid volume fraction in suspension $(-)$ \\
\hline$\omega$ & angular frequency $\left(\mathrm{s}^{-1}\right)$ \\
\hline
\end{tabular}

While most literature studies have measured velocity and attenuation variations in dilute suspensions $(<20$ vol. $\%)$, this study investigates variations in velocity, attenuation and mean frequency of ultrasonic signal for solids loading of up to $45 \mathrm{vol} . \%$. Moreover variations in the mixed particles systems are investigated for the first time. The particles used are 35,70 and $180 \mu \mathrm{m}$ glass beads and their mixtures selected to be representative of catalytic slurry reactors. It is often desired to determine the particle concentration and composition at different locations in a reactor. Calibration curves and procedure have been presented to determine particle concentration and size distribution.

\section{Experimental}

The experiments were conducted in a $0.1016 \mathrm{~m}$ diameter and $0.5 \mathrm{~m}$ tall Plexiglas cell equipped with a variable speed mechanical stirrer to maintain a homogeneous suspension of particles in liquid. The particles were glass beads of average diameter 35, 70 and $180 \mu \mathrm{m}$ and were suspended in tap water. A six-blade propeller ( $45^{\circ}$ pitch) of $0.03 \mathrm{~m}$ diameter provided the agitation and it was placed about $0.04 \mathrm{~m}$ from the column bottom. The ratio of suspension height to cell diameter was maintained around 1.3. Four baffles of $0.01 \mathrm{~m}$ width were also installed inside the cell. The rpm used for a given particle size and concentration was initially obtained from Zwietering [12] correlation for this type of setup. In order to ensure uniform suspension of particles, the rpm was increased above this initial value while measuring acoustic speed in the suspension. The rpm above which no change in acoustic speed was observed was selected for measurements. The final rpm (800-2000 range) for a given particle size and concentration was usually $10 \%$ or more above the calculated value. The temperature for the measurements was maintained at $25 \pm 0.1^{\circ} \mathrm{C}$ by circulating cold or hot water through a coil installed near the bottom of the cell. Two ultrasonic transducers were installed at $0.05 \mathrm{~m}$ above the bottom of the cell through two diametrically opposed ports about $0.02 \mathrm{~m}$ off the center. The ports allowed radial movement of the transducers. The radial distance used for measurements was $0.05 \mathrm{~m}$. A simplified sketch of the cell is shown in Fig. 1.

The ultrasonic system consisted of two $0.01 \mathrm{~m}$ diameter (LMN ceramic) ultrasonic transducers with a center frequency at $3 \mathrm{MHz}$ (50\% bandwidth) and a two channel

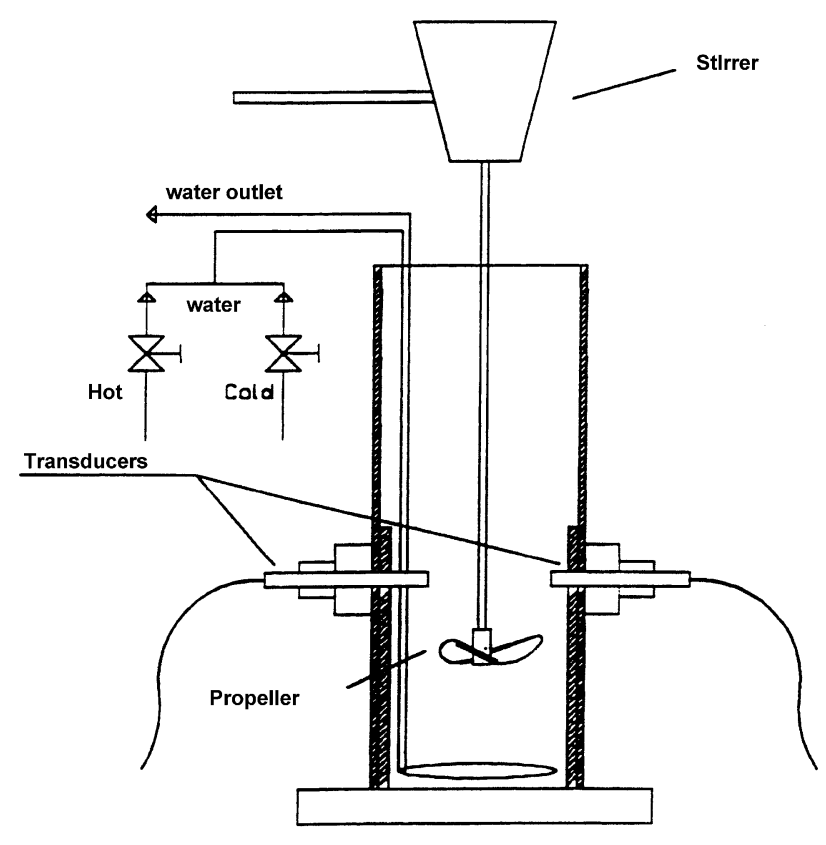

Fig. 1. Schematic diagram of the cell. 


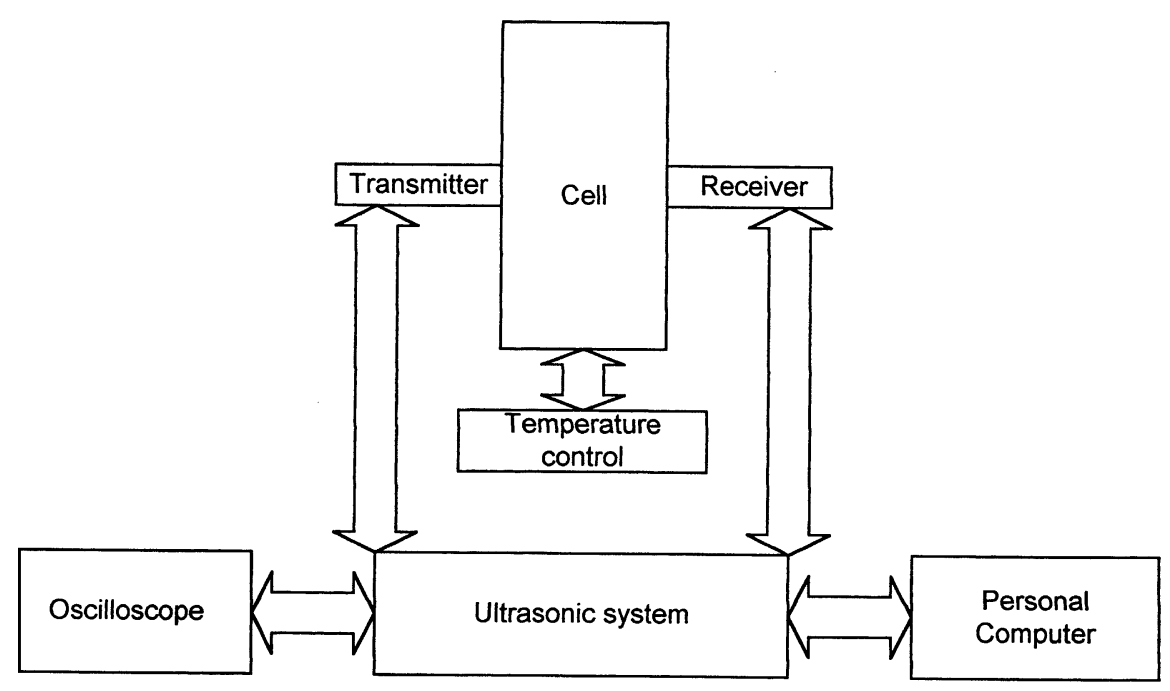

Fig. 2. Block diagram of the experimental setup.

ultrasonic pulse-receiver system designed for the frequency range $0.1-100 \mathrm{MHz}$. The system could be used either in pulse-echo mode or pitch-catch mode. In this study only pitch-catch mode was used. The system was modified to generate a relatively high energy pulse $(800 \mu \mathrm{J})$ for measurements in dense suspensions used in this study. All the system parameters, i.e. gain, pulse voltage, gate, energy were controlled via RS232 interface with a computer (Pentium $133 \mathrm{MHz}$ ).

A digital oscilloscope (TDS 210 from Tektronics) was used to visualize and analyze the received signal. In a typical experiment, after visualizing the signal on oscilloscope (avoiding signal saturation) a gate position and a gate width were selected according to the position of the signal. After an ultrasonic pulse was emitted by one of the transducers, the receiving transducer was activated at a time fixed by the gate delay and for the duration of selected gate width. During the time the gate was open the transmission time and the amplitude of the ultrasonic signal were measured. This process was repeated at a rate of $1 \mathrm{kHz}$ and a mean value was recorded at a rate of $1 \mathrm{~s}^{-1}$ Each plotted point is an average of 200 readings. Fig. 2 shows an integrated simplified block diagram of the overall experimental setup.

\section{Results and discussion}

\subsection{Variations of acoustic speed}

The effects of particle concentrations were measured up to about 45 vol. \%. The variations of acoustic speed with particle size and concentrations are presented in Fig. 3 based on average of up to six replicates. The standard deviation from these replicates was less than $0.1 \%$ indicating a good reproducibility of the results. The change in velocity is generally less significant below solids concentration of about 5 vol.\% but increases for higher solids concentrations. Fig. 3 also shows that for the same solid concentration, acoustic speed decreased with increasing particle size. Fig. 4 compares the data of this study for $35 \mu \mathrm{m}$ particles with the results of Atkinson and Kytomaa [13] obtained with particles of similar wavenumber $(k r \approx 0.2)$. It can be seen that there is good agreement between the two sets of data from these independent studies.

In suspensions of fine particles with small wavenumber $(k r \ll 1)$, the medium can be treated to be homogeneous and the phenomenological approach by Urick [6] would prove

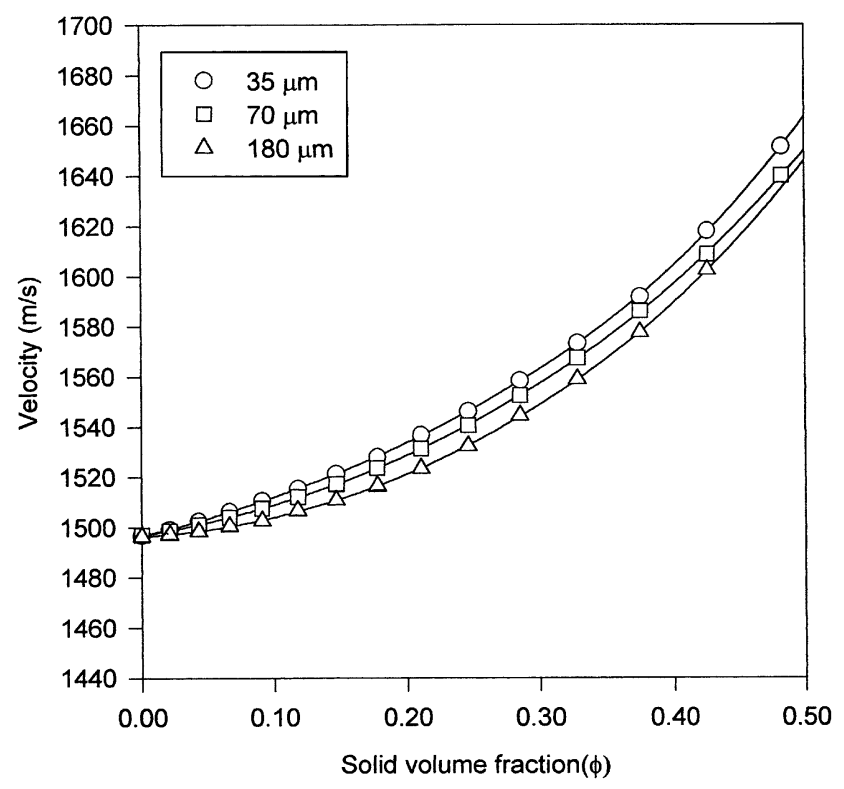

Fig. 3. Variation of acoustic velocity with solid concentration in water-glass bead slurries of different particle sizes. 


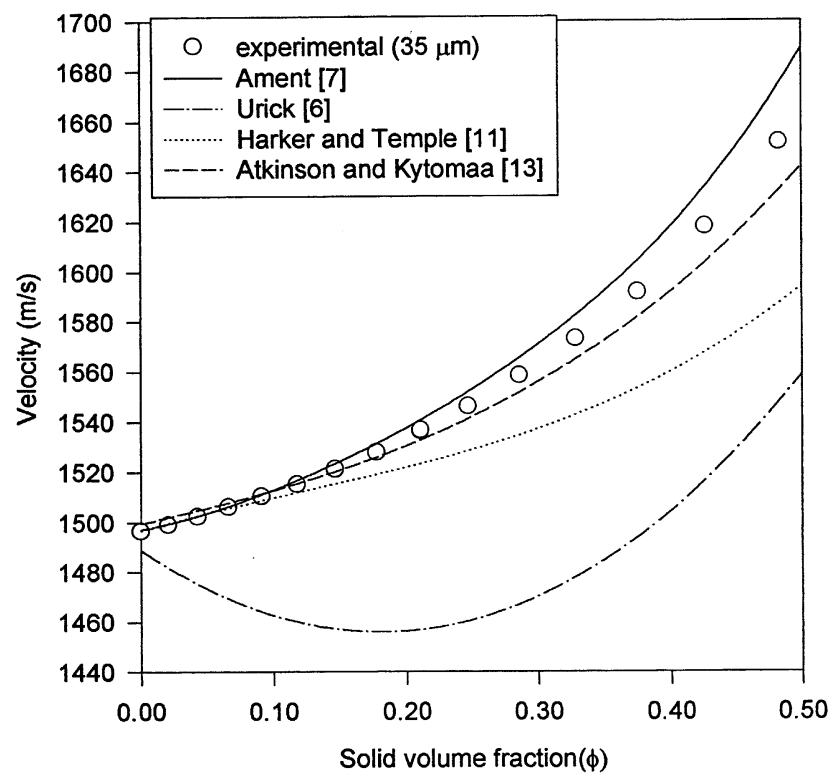

Fig. 4. Comparison of experimental acoustic velocity with literature data and theory.

useful $[13,14]$.

$V=\frac{1}{\sqrt{\rho_{\text {eff }} \beta_{\text {eff }}}}$

where $\rho_{\text {eff }}, \beta_{\text {eff }}$ are the average values of density and compressibility of the mixture;

$\rho_{\text {eff }}=\rho_{\mathrm{s}} \phi+\rho_{\mathrm{f}}(1-\phi)$

$\beta_{\text {eff }}=\beta_{\mathrm{s}} \phi+\beta_{\mathrm{f}}(1-\phi)$

Large differences found between experimental results and calculated values by Urick's formula (Fig. 4) can be attributed to relatively large values of $k r(0.2-1)$ used in this study. Improvements to Urick's equation have been proposed [7] and found useful in describing the observed behavior of acoustic velocity by several investigators $[11,15]$. The improvements have been obtained by including the effects of fluid viscosity and particle size in the effective density expression and thus taking into account the heterogeneous nature of the medium. Harker and Temple [11] analyzed the propagation of an acoustic wave through a mixture of two phases using the hydrodynamic equations to balance momentum and continuity of the phases with the drag of one phase on another. They assumed that there was no heat or mass transfer between phases and no gravitational field. Their final equation can be rewritten as a complex wave vector $k$ of the form [16]

$k_{1}^{2}=\omega^{2} \beta_{\mathrm{eff}} \rho_{\mathrm{eff}}(\omega)$

where

$\rho_{\text {eff }}(\omega)=\frac{\rho_{\mathrm{f}} \rho_{\mathrm{s}}+\left[\phi \rho_{\mathrm{s}}+(1-\phi) \rho_{\mathrm{f}}\right] M(\omega)}{M(\omega)+\left[\phi \rho_{\mathrm{f}}+(1-\phi) \rho_{\mathrm{s}}\right]}$
The real part of the complex wave vector $k$ gives the velocity of the propagated wave, while the imaginary part describes the wave attenuation. Harker et al. [16] compared several existing theoretical models by simply replacing $M(\omega)$ with the corresponding expressions developed by other researchers. The expressions by Ament [7] and Harker and Temple [11] account for the particle size effects and were tested against the data of this study. As shown in Fig. 4, the fit is good for $35 \mu \mathrm{m}$ particles with the Ament's equation over the whole range of solids concentrations while the approach by Harker and Temple deviates quite significantly above 12 vol. $\%$. The effect of particle diameter was not predicted properly by these equations. The data of this study shows a decrease in acoustic velocity with increasing particle size while predictions show an increase in acoustic velocity with increasing particle size. This can be attributed to relatively low values of Wavenumber $(k r<0.1)$ for which these theoretical equations are applicable. The particle Wavenumber for the smallest particles used in this study is about 0.2 which is close to the theoretical limit where the predictions are reasonable.

For practical purposes, the variation of acoustic velocity with particles concentration can be correlated by modified form of Urick's equation as proposed by Pinfield et al. [17]:

$\frac{1}{V^{2}}=\frac{1}{V_{0}^{2}}+\beta_{1} \phi+\beta_{2} \phi^{2}$

where $\beta_{1}$ and $\beta_{2}$ are experimentally determined constants which are functions of particle size. Providing good temperature and mixing controls are ensured, calibration lines can be constructed in this manner for different industrial setups. Fig. 5 shows calibration lines for the three particle sizes used in the present study.

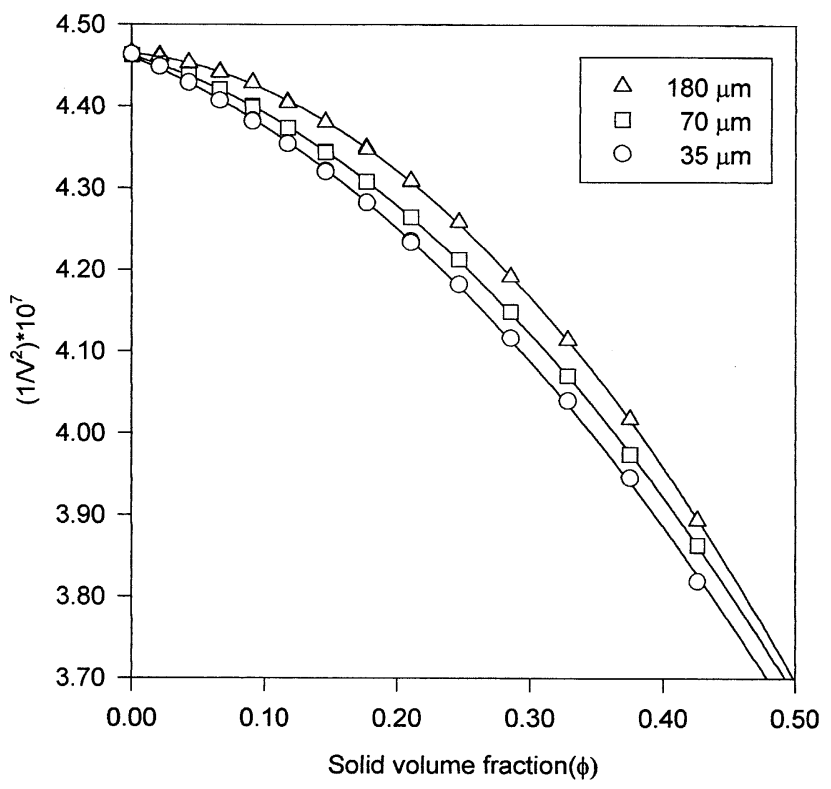

Fig. 5. Calibration curves for acoustic velocity versus solid volume fraction for different particle sizes. 
$35 \mu m$ particles:

$\frac{1}{V^{2}}=4.46-0.176 \phi-2.725 \phi^{2}, \quad R^{2}=0.99$

$70 \mu$ m particles:

$\frac{1}{V^{2}}=4.46-0.483 \phi-2.163 \phi^{2}, \quad R^{2}=0.99$

$180 \mu m$ particles:

$\frac{1}{V^{2}}=4.46-0.656 \phi-1.95 \phi^{2}, \quad R^{2}=0.99$

Such calibration curves can be used to determine slurry concentration from the measurement of acoustic velocity in suspensions of known particle size.

Measurements were also conducted in suspensions of mixed particle sizes of glass beads-water system. Two sets of experiments were conducted by adding glass beads of 70 or $180 \mu \mathrm{m}$ in a $15 \mathrm{vol} . \%$ suspension of $35 \mu \mathrm{m}$ particles. The objective was to observe the sensitivity as well as test the additive effect on velocity with increasing fraction of larger particles. As seen in Fig. 6, the curves for mixed particle suspensions deviate from the curve of the $35 \mu \mathrm{m}$ particles with increasing fraction of larger particles. The velocity data for single and mixed particles suspensions were plotted as a function of an average dimensionless wavenumber defined as

$k r_{\mathrm{avg}}=k\left[r_{1} \varphi+r_{2}(1-\varphi)\right]$

where $\varphi$ is a fraction of larger particle size in total solids in the suspension. It can be seen in Fig. 7 that for the same overall solids concentration but different mass fraction of larger particles, the measured velocibies are between those

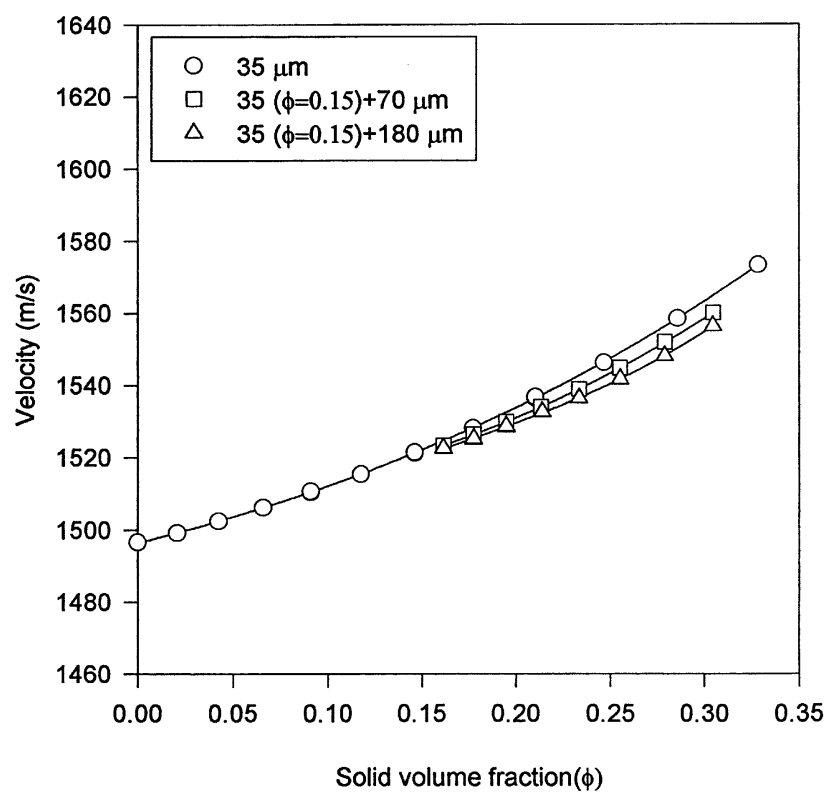

Fig. 6. Acoustic velocity versus solid concentration in water-glass bead systems of mixed particle sizes.

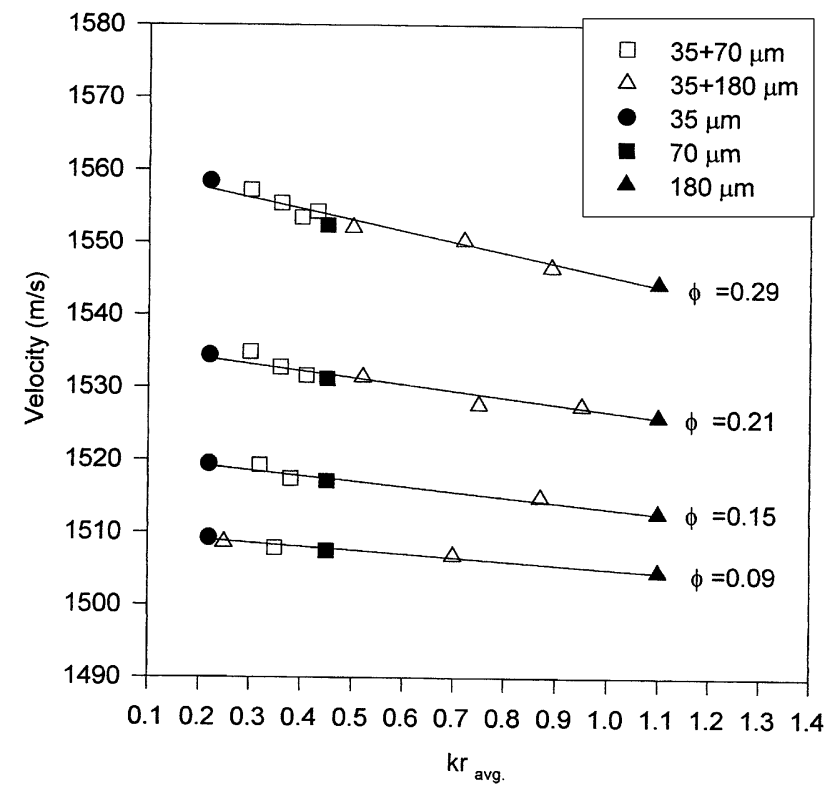

Fig. 7. Acoustic velocity as a function of dimensionless wavenumber with slurry concentration as parameter.

obtained with the particles of the two sizes in mixture. This shows an additive effect of the particles size and can be a basis to determine fraction of each particle in a mixture of known total concentration and particle sizes. This approach, however, may be limited to mixtures of two particle sizes only.

\subsection{Attenuation measurements}

Attenuation of an acoustic wave passing through a medium provides additional information about the composition of the medium. The attenuation coefficient $\left(\alpha_{i}\right)$ defined by Eq. (8) was calculated for each incremental addition of particles,

$\alpha=\sum_{i=1}^{n} \alpha_{i}=-\sum_{i=1}^{n} \frac{1}{L} \ln \left(\frac{A_{i}}{A_{o i}}\right)$

Here $A_{o i}$ represents the amplitude level before incremental solids addition and $A_{i}$ after addition. The attenuation for a given total solids concentration was calculated by summing the incremental attenuations. This procedure was adopted to avoid saturation of signal at low solids concentrations and to account for the increase in gain required at higher solids concentrations for the signal to be received. Fig. 8 presents attenuation coefficients obtained with the increasing solid fraction for different particle sizes. It can be observed that attenuation increases with increasing particle size and concentration. The rate of increase, however, varies with particle size, being higher for larger particles. For the largest particles, the change in attenuation coefficient with increasing solid concentration becomes less significant above about 15 vol.\%. As $k r$ increases at the same 


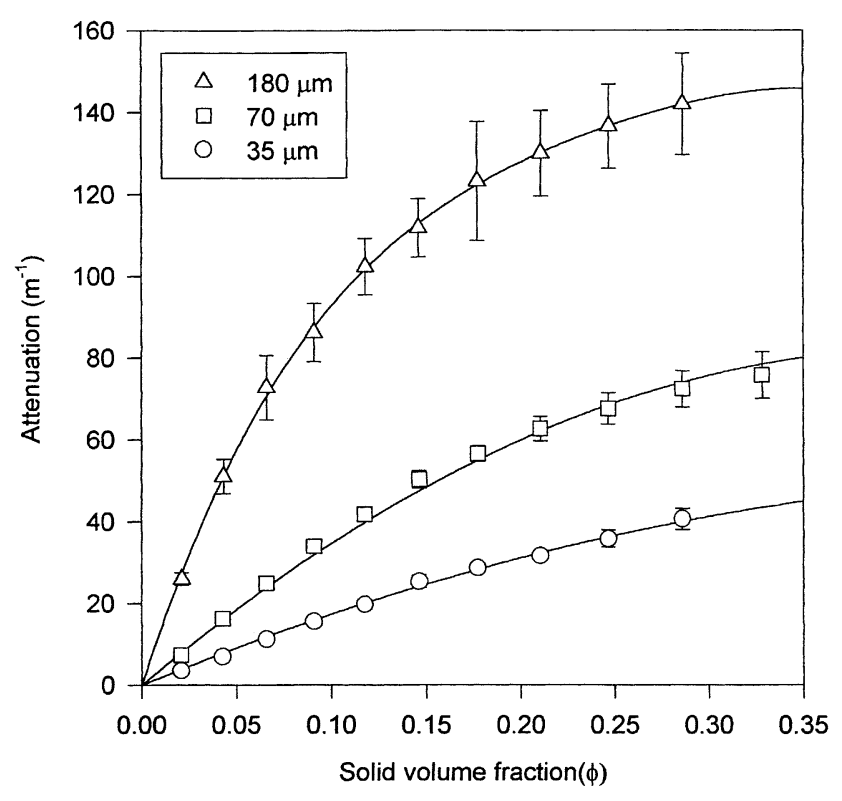

Fig. 8. Measured attenuation coefficient as a function of slurry concentration. Error bars show \pm S.D.

applied frequency (i.e. as the particle size increases), the multiple scattering phenomena becomes more important causing a deviation of the overall attenuation from linear behavior for the largest particle size (Fig. 8). Attenuation of ultrasonic signal in liquid-solid suspensions has been reported by several researchers $[9,10,13]$. Atkinson and Kytomaa [18] observed the attenuation to be a function of both applied frequency and solids concentrations. The increase in attenuation was almost monotonic up to about $20 \mathrm{vol} \%$, solid concentration being more prominent at higher $k r>0.75$. Attenuation in liquid-solid suspensions could be estimated by the approach originally developed by Epstein [19] for elastic solids and subsequently verified by Stakutis et al. [9] for other types of solids. The total attenuation coefficient as derived by Epstein consisted of three parts:

$\alpha_{\mathrm{P}}=\alpha_{\mathrm{S}}+\Delta \alpha_{\mathrm{V}}+\alpha_{\mathrm{V}}$

where $\alpha_{\mathrm{S}}$ is the attenuation caused by scattering phenomena, $\alpha_{\mathrm{V}}$ the absorption caused by viscous drag at the particle surface and $\Delta \alpha_{\mathrm{V}}$ the correction of the absorption for larger $k r$. Epstein and Carhart [8] used a thermal absorption term as well to fully characterize the absorption of the acoustic wave. However, for suspensions where the particle density is more than twice that of the suspending medium, thermal conduction losses are very small compared to viscous drag losses $[10,19]$. Indeed, we calculated $\alpha_{\mathrm{H}}$ from the theoretical equations of Allegra and Hawley [10] and the resulting values were very small. That is why this term is not accounted for in this work. The attenuation coefficient $\alpha_{\mathrm{P}}$ was calculated by the following equation from

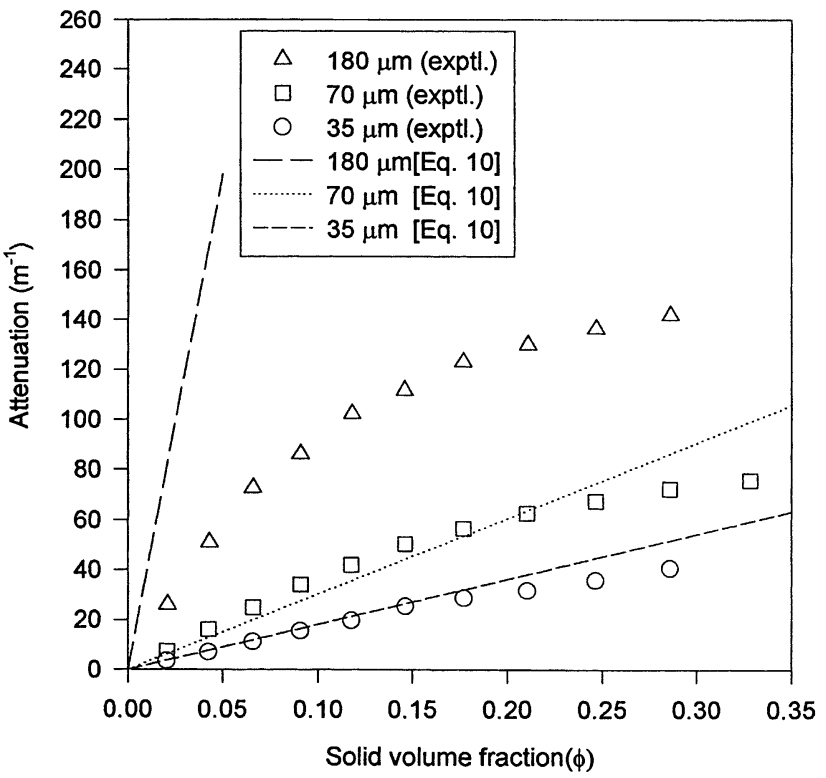

Fig. 9. Comparison of theoretical and experimental attenuation for different particle sizes.

Stakutis et al. [9]:

$$
\begin{aligned}
\alpha_{\mathrm{P}}= & \phi k(\delta-1) \operatorname{Re}\left[\frac{i+b-i b^{2} / 3}{\delta-i \delta b-(2+\delta) b^{2} / 9}\right] \\
& +\frac{1}{3} \phi k^{3} r^{2} \operatorname{Re}\left[\frac{10}{9 b}+\frac{23^{i}}{4 b^{2}}\right]+\frac{1}{3} \phi k^{4} r^{3} \\
& \times\left[\left(\frac{\delta-1}{\delta-2}\right)^{2}+\frac{1}{3}\left(1-\frac{3 B}{3 \gamma+2 \mu}\right)^{2}\right]
\end{aligned}
$$

where $\eta$ is the fluid viscosity, $\delta=\rho_{\mathrm{f}} / \rho_{\mathrm{s}}, b=\left[\left(i \omega \rho_{\mathrm{f}} / \eta\right)^{0.5} r\right]$, and $\gamma$ and $\mu$ are Lamé elastic constants of the particles. The calculated attenuations obtained with the above equation are shown in Fig. 9 for different particles and concentrations. The predictions are good for 35 and $70 \mu \mathrm{m}$ particles up to solids fraction of about 0.25 . For the $35 \mu \mathrm{m}$ particles, the calculated viscous term provided the main contribution while for $70 \mu \mathrm{m}$ particles, scattering also became important. The experimental values obtained with $180 \mu \mathrm{m}$ particles could not be predicted by the above equation. This could be attributed to high Wavenumber for this particle size $(>1)$ where the above equation may not apply. Attenuation measurements with particles of large Wavenumbers will be the subject of a future study.

Attenuation measurements provide another means of obtaining particles concentration in a suspension of known particle size and could be used to verify the composition of the suspension obtained from velocity measurements. This will be especially significant for slurry concentrations below $10 \mathrm{vol} . \%$ where the changes in attenuation are more sensitive than variations in acoustic velocity. Attenuation measurements were also conducted in mixed particles 

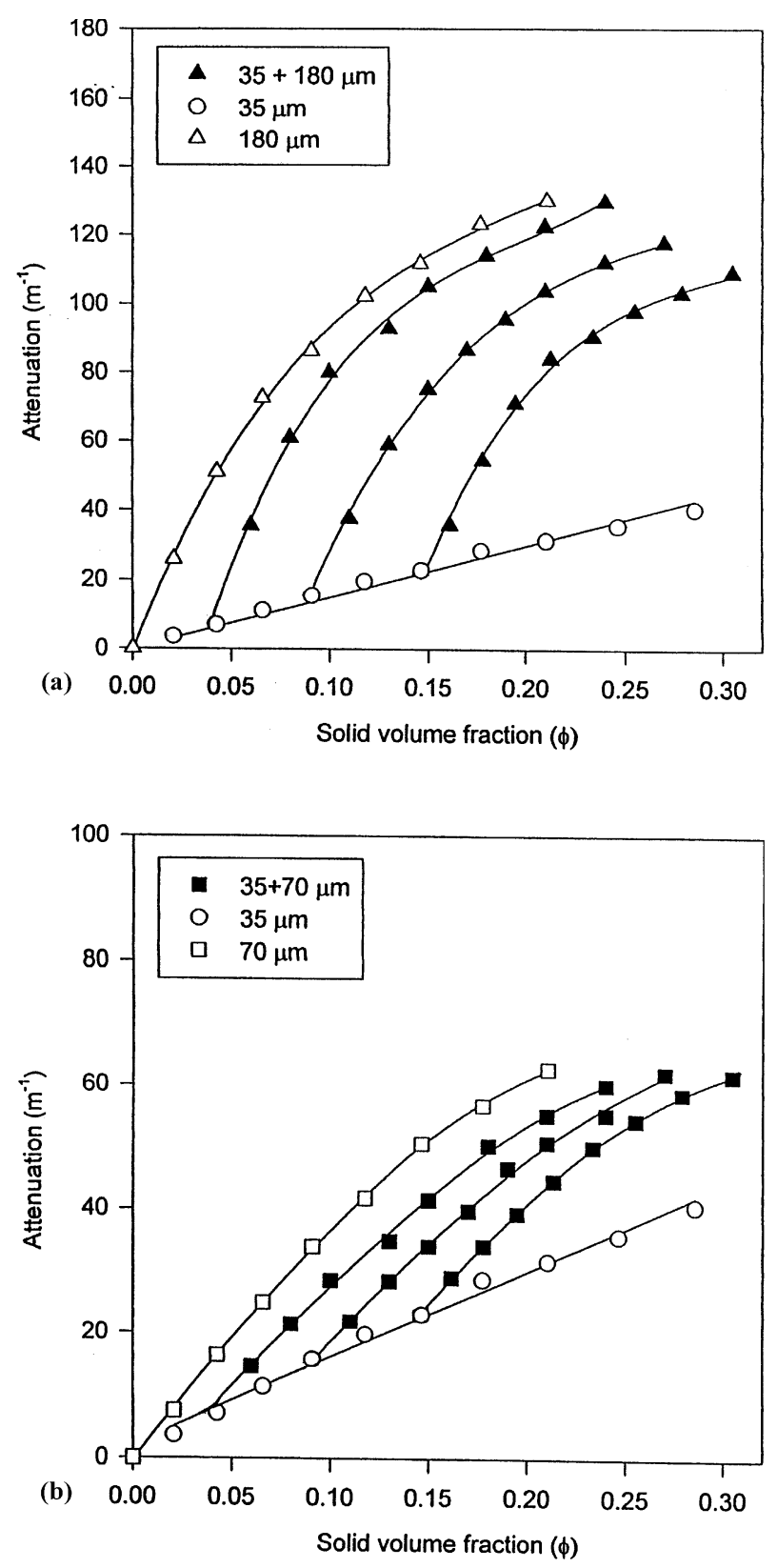

Fig. 10. Comparison of attenuation obtained in suspensions of single and mixed particles suspensions.

suspensions. Fig. 10a and $\mathrm{b}$ shows changes in attenuation with increasing concentration of 180 and $70 \mu \mathrm{m}$ particles in suspensions containing different initial concentrations of $35 \mu \mathrm{m}$ particles. It can be seen that the addition of larger particles in a suspension of $35 \mu \mathrm{m}$ particles increases the attenuation nearly proportional to the concentration of larger particles. The curves for mixed particles are nearly parallel to the curve for larger particles. The rate of increase is fast at low slurry concentrations but slows down at higher slurry concentrations. Fig. 11 is a plot of dimensionless Wavenumber as a function of attenuation with slurry concentration as a parameter. The average value of ' ' $k r$ ' for binary mixtures of solids (from Fig. 10a and b) were

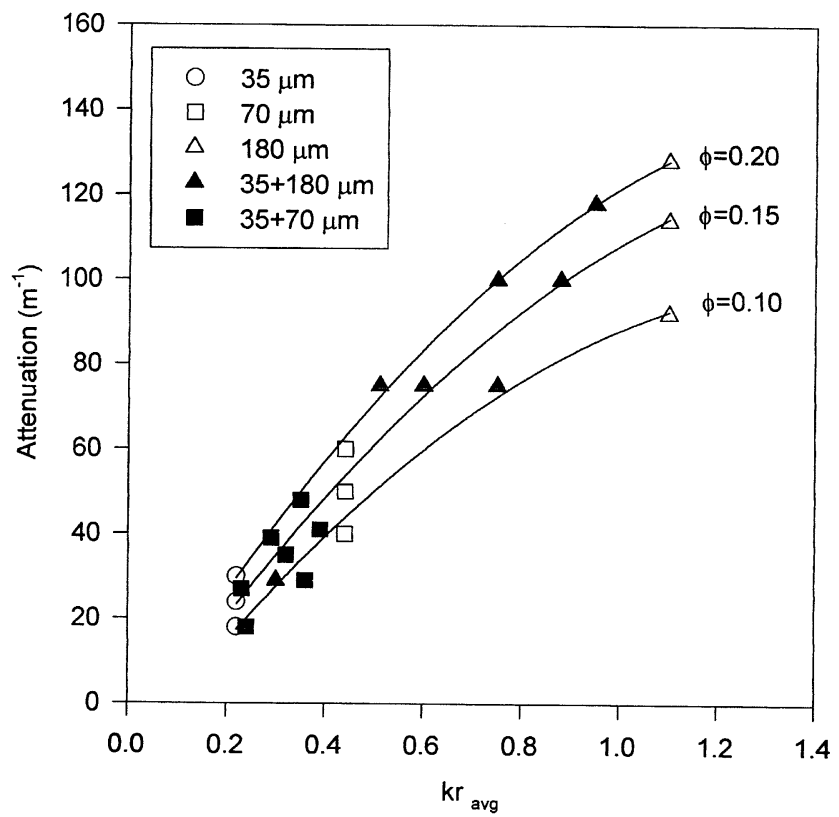

Fig. 11. Measured attenuation as a function of dimensionless wavenumber for different solid fractions.

calculated using Eq. (7). Such a plot can be used to verify the deductions from velocity measurements. An increase in the concentration of larger particles (say due to agglomeration process) in a suspension can be traced along the curves of known slurry concentration.

\subsection{Variations of peak frequency}

The liquid-solid suspensions were further characterized by analyzing the captured wave form. The peak frequency obtained by Fourier transform was plotted as a function of solid volume fraction. The peak frequency was calculated by averaging 10 values given by the oscilloscope over a period of time. The standard deviation of these readings was found to be less than $2 \%$. A change in peak frequency was observed only for larger particles (70 and $180 \mu \mathrm{m})$ while for the smallest particles $(35 \mu \mathrm{m})$ there was no significant effect on the peak frequency (approximately same as in the water). There is a direct proportionality between solid concentration and the measured peak frequency of the acoustic pulse for both 70 and $180 \mu \mathrm{m}$ glass bead particles (Fig. 12). From the analysis of attenuation measurements, it was observed that the energy loss in the suspension of $35 \mu \mathrm{m}$ particles $(k r=$ 0.2 ) could be attributed to viscous dissipation while scattering became important for larger particles. Therefore when viscous dissipation dominates (i.e. $k r<0.2$ ), the peak frequency does not get altered significantly. This property can be utilized to detect increase in particle size in a suspension due to such processes as agglomeration or crystallization. Based on these observations some experiments were conducted to measure the fraction of particles of larger size (70 or $180 \mu \mathrm{m})$ in a liquid-solid system where the solid phase 


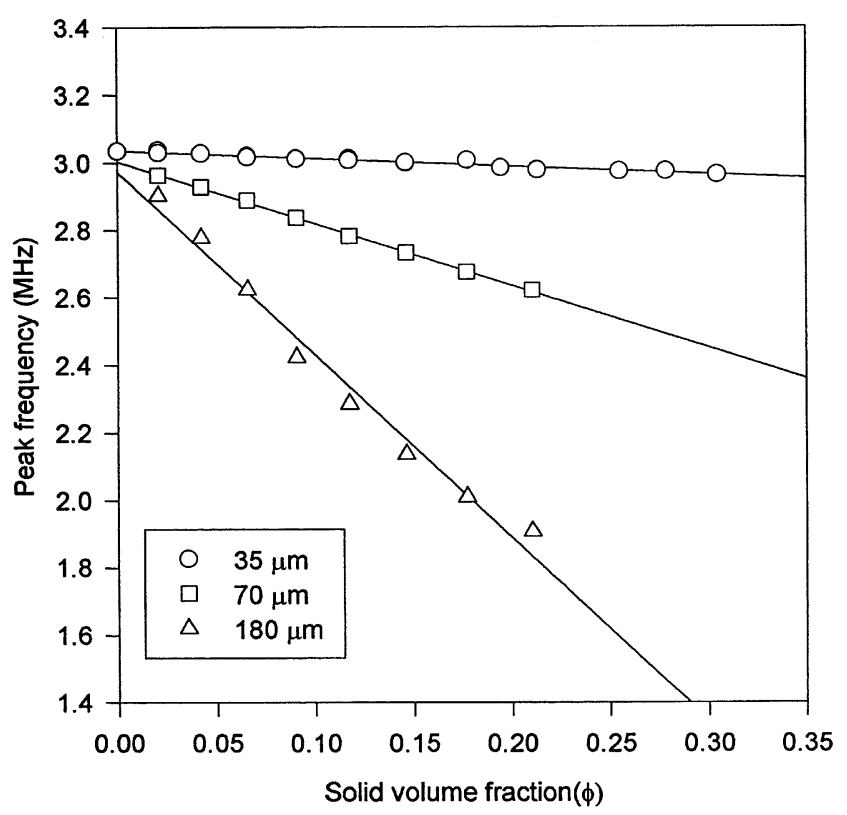

Fig. 12. Peak frequency of acoustic peak as a function of solid concentration for different particle sizes.

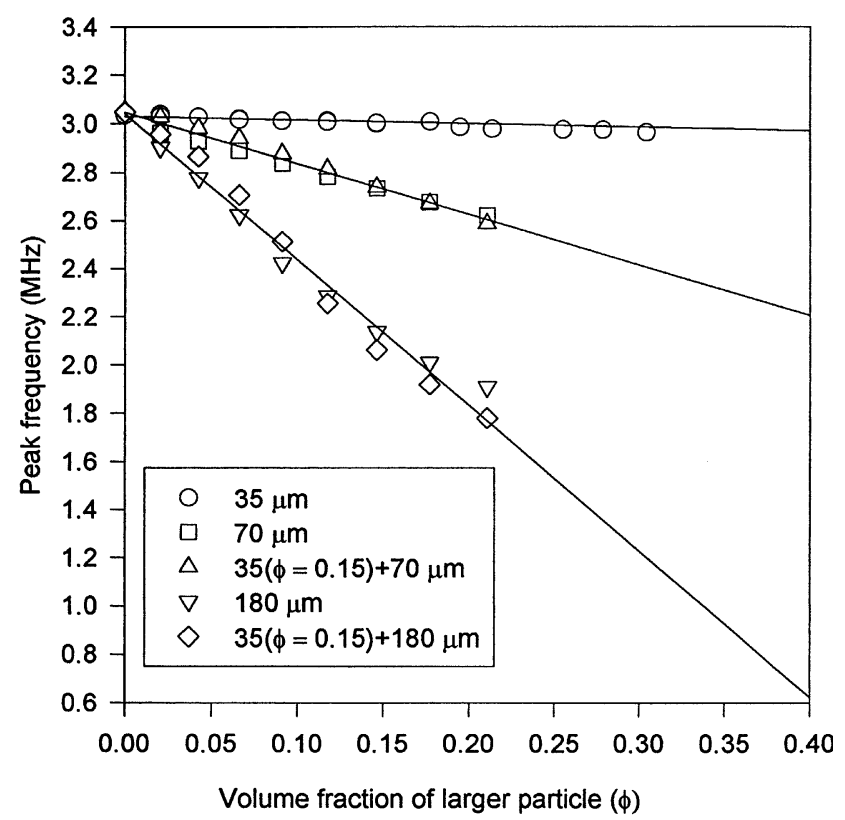

Fig. 13. Comparison of peak frequencies in single and mixed particles systems.

was a mixture of $35-70$ or $35-180 \mu \mathrm{m}$ glass beads. The results obtained with mixed particles were compared with those obtained when only one particle size was suspended in water at the corresponding concentrations (Fig. 13). It can be seen that there is a good overlap indicating that the presence of $35 \mu \mathrm{m}$ particles did not significantly affect the variation in peak frequency due to larger particles in the system. This indicates that an increase in particle size could be detected in a suspension by this technique as well. This approach could also be extended to determine particle size distribution in a suspension with the help of a variable frequency ultrasonic system. The solution of this inverse problem could, however, encounter problems discussed by Spelt et al. [20]. These and other related issues will be the subject of a future study.

\section{Conclusions}

The usefulness of ultrasonic techniques are demonstrated for the characterization of dense slurries including slurries of mixed particle sizes. The variation of acoustic velocity with slurry concentration is quite significant in the range $10-50$ vol.\% solids. For lower slurry concentrations, the variations of attenuation and peak frequency (when applicable) are more significant. The average particle size in a suspension of known slurry concentration could be determined from the velocity measurements and plots of ' $k r$ ' as a function of velocity with slurry concentration as a parameter. The trends in particle size variations in a suspension could be traced with attenuation measurements. Growth in particle size above a certain value could be detected by changes in peak frequency of the acoustic pulse.

\section{References}

[1] S.J. Hwang, L.S. Fan, Chem. Eng. J. 33 (1986) 49.

[2] A. Yasunishi, M. Fukuma, K. Muroyama, J. Chem. Eng. Japan. 29 (1986) 444.

[3] L.S. Fan, T. Yamashita, R.H. Jean, Chem. Eng. Sci. 42 (1987) 17-25.

[4] S.L.P. Lee, H.I. de Lasa, AIChE J. 33 (1987) 1359-1370.

[5] M.S. Grenwood, J.L. Mai, M.S. Good, J. Acoust. Soc. Am. 94 (1993) 908-916.

[6] R.J. Urick, J. Appl. Phys. 18 (1947) 983-987.

[7] W.S. Ament, J. Acoust. Soc. Am. 25 (1953) 638.

[8] P.S. Epstein, R.R. Carhart, J. Acoust. Soc. Am. 25 (1953) 553-565

[9] V.J. Stakutis, R.W. Morse, M. Dill, R.T. Beyer, J. Acoust. Soc. Am. 27 (1955) 539-546.

[10] J.R. Allegra, S.A. Hawley, J. Acoust. Soc. Am. 51 (1971) 1545-1563.

[11] A.H. Harker, J.A.G. Temple, J. Phys. D 21 (1987) 1576-1588.

[12] T.N. Zwietering, Chem. Eng. Sci. 8 (1957) 244.

[13] C.M. Atkinson, H.K. Kytomaa, Int. J. Multiphase Flow 18 (1992) 577-592.

[14] L. Hampton, J. Acoust. Soc. Am. 42 (1967) 882-890.

[15] D.J. McClements, M.J.W. Povey, Adv. Colloid Interf. Sci. 27 (1987) 285-316.

[16] A.H. Harker, P. Schofield, B.P. Stimpson, R.G. Taylor, J.A.G. Temple, Ultrasonics 29 (1991) 427-438.

[17] V.J. Pinfield, M.J.W. Povey, E. Dickinson, Ultrasonics 33 (1995) 243-251.

[18] C.M. Atkinson, H.K. Kytomaa, J. Fluid. Eng. 115 (1993) 665.

[19] P.S. Epstein, Applied Mechanics, Theodore von Karman Anniversary Volume, California Institute of Technology, Pasadena, 1941, p. 162.

[20] P.D.M. Spelt, M.A. Norato, A.S. Sangani, L.L. Tavlarides, Phys. Fluid. 11 (1999) 1065 